\title{
A fast-healing and high-performance metallosupramolecular elastomer based on pyridine-Cu coordination
}

\author{
Hui Liu, Qiaoqiao Shen ${ }^{1}$, Linjun Zhang ${ }^{1}$, Shiyu Gu${ }^{1}$, Yan Peng ${ }^{1}$, Qi Wu ${ }^{1}$, Hui Xiong ${ }^{1}$, Hao Zhang ${ }^{1}$, \\ Lijuan $\mathrm{Zhao}^{2}$, Guangsu Huang ${ }^{1}$ and Jinrong $\mathrm{Wu}^{1^{*}}$
}

\begin{abstract}
Elastomers easily undergo a catastrophic failure as soon as a crack is introduced by mechanical damage. Thus, it is extremely important for elastomers to possess fast healing ability, which enables the quick reparation of cracks. However, developing elastomers with fast self-healing ability and high mechanical strength is highly challenging. Herein, we fabricate a metallosupramolecular elastomer by facilely introducing pyridine-Cu coordination into a copolymer of ethyl acrylate and vinyl pyridine. Interestingly, the pyridine- $\mathrm{Cu}$ coordination has a strong photothermal effect, which readily increases the sample temperature to $60^{\circ} \mathrm{C}$ in $30 \mathrm{~s}$ under near-infrared light. At this temperature, the sticky reptation modes are activated and thus serve as the driving force for network reorganization and fast self-healing of the metallosupramolecular elastomer. Albeit with a tensile strength of $10 \mathrm{MPa}$, the scratched and completely fractured samples can be healed within $2 \mathrm{~min}$ and $3 \mathrm{~h}$, respectively. Moreover, during the damage and healing processes, the break and reformation of the coordination bonds can be tracked through laser confocal micro-Raman spectroscopy. This provides a microscopic methodology to monitor the bond-level healing kinetics of metallosupramolecular polymers.
\end{abstract}

Keywords: metallosupramolecular elastomer, photothermal effect, molecular dynamics, healing mechanism, laser confocal micro-Raman

\section{INTRODUCTION}

The self-healing ability of creatures has inspired the rapid development of biomimetic artificial materials that can repair mechanical damages with or without the assistance of external intervention. To endow artificial materials with intrinsic selfhealing ability, it is necessary to construct dynamic networks formed by either dynamic covalent or noncovalent bonds [1-9]. These dynamic bonds can break and reform, enabling the reorganization and restoration of dynamic networks at the damage site. Thus far, much effort has been devoted to developing mechanically robust self-healing materials [3,10-14]. However, the materials usually take several hours or days to restore their mechanical properties. In fact, the healing speed is as important as the mechanical strength [15-19]. In many applications, such as space shuttle, airplane, high-speed train, and heavy-duty vehicle, once a crack appears, a catastrophic accident may occur if it is not repaired in time. Therefore, it is extremely important to develop fast self-healing materials without compromising their mechanical properties.

Among the diverse dynamic bonds to construct self-healing materials, metal-ligand coordination is highly tunable by selecting different combinations of metal ions and ligands. Therefore, its strength can be adjusted from $25 \%$ to $75 \%$ bond energy of covalent bonds [20]. Moreover, many metal-ligand coordination interactions have a light-responsive property. Burnworth et al. [21] first exploited metal-ligand coordination to fabricate a metallosupramolecular polymer that could be repaired through exposure to ultraviolet (UV) light. As the metal-ligand absorbed the UV light, the surface temperature of the polymer increased, which promoted the dissociation of ligand-metal binding on the crack surfaces. When the UV light was turned off, new bonds were formed upon cooling the sample. Taking advantage of the light-responsive property, the repair process can be conducted on demand by shedding a light stimulus, making it feasible for long-distance repair and selected area healing [22]. However, the healing process cannot be accomplished in a short time for most reported metallosupramolecular polymers $[2,13,23]$.

In addition to the development of self-healing polymers, understanding the multilevel structural evolution during the self-healing process is also crucial. To evaluate the self-healing process, optical microscopy [24], atomic force microscopy [21], super depth of field three-dimensional microscopy [25], scanning electron microscopy [26] and confocal laser scanning microscopy [27] are usually used to monitor the closure of scratches. These microscopy methods can provide quantitative and visualizable information for the healing process. To further study the self-healing process in the bond level, spectrum analysis, such as Fourier transform infrared (FTIR) spectroscopy [28], Raman spectroscopy [29] and dielectric spectroscopy [30] are used. Geitner et al. [29] monitored a thiol-ene reversible reaction on scratches and their edge areas by molecular antiStokes Raman scattering microscopy. Davydovich et al. [31] used through-space two-dimensional nuclear magnetic resonance (NMR) measurements and FTIR analysis to monitor the change in van der Waal interactions during the self-healing process of a copolymer. It was revealed that water accelerated the damage repair of hydrophobic copolymers. Despite these

\footnotetext{
${ }^{1}$ State Key Laboratory of Polymer Materials Engineering, College of Polymer Science and Engineering, Sichuan University, Chengdu 610065, China

${ }^{2}$ College of Chemistry and Materials Science, Sichuan Normal University, Chengdu 610066, China

* Corresponding author (email: wujinrong@scu.edu.cn)
} 
significant advancements, it remains a challenge to track the molecular-level dynamics and bond breakage-reformation around the damage sites.

Here, we develop a novel metallosupramolecular elastomer with high mechanical strength and fast self-healing ability and investigate the molecular-level driving force and bond-level network reorganization for the self-healing process. Specifically, the metallosupramolecular elastomer is prepared by simply copolymerizing ethyl acrylate (EA) and vinyl pyridine (VP) and then coordinating them with cupric ions (Fig. 1a). Experiments demonstrate the strong photothermal effect of pyridine- $\mathrm{Cu}$ coordination. The photothermal effect activates the molecular motion of sticky reptation modes, which acts as the driving force to promote the reformation of dissociated coordination interaction induced by mechanical damage. As such, the damaged elastomer can be readily healed within a short time. Interestingly, the break and reformation of the pyridine- $\mathrm{Cu}$ coordination can be monitored by laser confocal Raman microscopy (Fig. 1b), which enables the real-time monitoring of bond-level healing kinetics, and therefore allows us to have a better understanding of the underlying damage-restoring mechanism.

\section{EXPERIMENTAL SECTION}

\section{Synthesis}

An EA-VP copolymer was synthesized by a free-radical reaction between EA and VP. EA-VP-M $\mathrm{M}_{x}$ was prepared by adding a metal chloride solution into EA-VP, where $\mathrm{M}$ denotes the metal ions including $\mathrm{Cu}^{2+}, \mathrm{Co}^{2+}$, and $\mathrm{Zn}^{2+}$, and $x$ represents the molar ratio of $M$ to pyridine. The film was formed by the film casting method.

An ethylpyridine-Cu complex was prepared by adding an ethanol solution of copper chloride into ethylpyridine. The obtained blue crystals were entirely washed with ethanol and dried in a vacuum.

\section{Characterization}

${ }^{1} \mathrm{H}-\mathrm{NMR}$ spectra were recorded using an $\mathrm{HD} 400-\mathrm{MHz}$ spectrometer (Bruker), and $\mathrm{CD}_{3} \mathrm{OD}$ was used as a solvent. Tensile tests and loading-unloading tests were conducted at room temperature on an electronic tensile machine with a strain rate of $100 \mathrm{~mm} \mathrm{~min}$. The molecular weight of the copolymer was measured using Tosoh HIC-8320GPC with tetrahydrofuran as an eluent. Micro-Raman spectra were obtained using a LabRAM HR Evolution micro-Raman spectrometer (HORIBA Jobin Yvon) equipped with 532-, 633-, and 785-nm laser sources. Rheological properties were recorded using HAAKE MARS III. Dynamic mechanical properties were measured using Q800 (TA Instruments) in a tension mode. Differential scanning calorimetry (DSC) measurements were examined on Q2000 (TA Instruments) within the temperature range of -70 to $100^{\circ} \mathrm{C}$ at

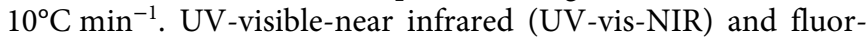
escence spectra were obtained on a SHIMADZU UV-2600 U spectrophotometer and a fluorescence spectrometer (FluoroMax-4), respectively. The online tracing for the recovery of the scratch was performed on a polarizing microscope (Eclipse LV100N POL, Nikon) equipped with a heating stage. The surface temperature of the sample was recorded on an infrared thermal image instrument (FLIR T420) using a video mode.

\section{RESULTS AND DISCUSSION}

\section{Preparation of the metallosupramolecular elastomer}

To fabricate a metallosupramolecular elastomer, EA and VP were firstly copolymerized by simple radical initiation (Fig. 1a and Fig. S1). The resulting copolymer denoted as EA-VP has an average molecular weight $\left(M_{\mathrm{n}}\right)$ of $85.0 \mathrm{~kg} \mathrm{~mol}^{-1}$ as measured by gel permeation chromatography. This molecular weight is higher than the critical entanglement molecular mass $\left(26.0 \mathrm{~kg} \mathrm{~mol}^{-1}\right)$ of PEA [32], indicating that the EA-VP molecules are entangled. To crosslink the EA-VP molecules with metal ions, it is dissolved in ethyl acetate. Subsequently, the methanol solution containing metal chloride is slowly added into the as-prepared EA-VP solution. Next, the resulting solution is cast in the metallosupramolecular elastomer EA-VP-M $\mathrm{M}_{x}$. The mechanical properties of EA-VP-M $\mathrm{M}_{x}$ are shown in Fig. S2.

To examine the photothermal effect of EA-VP and EA-VP-M with different metal ions, we used an infrared camera to monitor the temperature profile during the 785-nm laser-on and laser-off processes. The laser power is $1.4 \mathrm{~W} \mathrm{~cm}^{-2}$, and the thickness of EA-VP-M $\mathrm{M}_{x}$ film is $0.63 \mathrm{~mm}$. When the laser is shed on the EAVP film for $2 \mathrm{~min}$, the surface temperature of the irradiation point shows a subtle change from 24 to $26^{\circ} \mathrm{C}$ (Fig. 2a). Nevertheless, as to the EA-VP-Cu $\mathrm{Cu}_{1 / 12}$ film, the surface temperature of

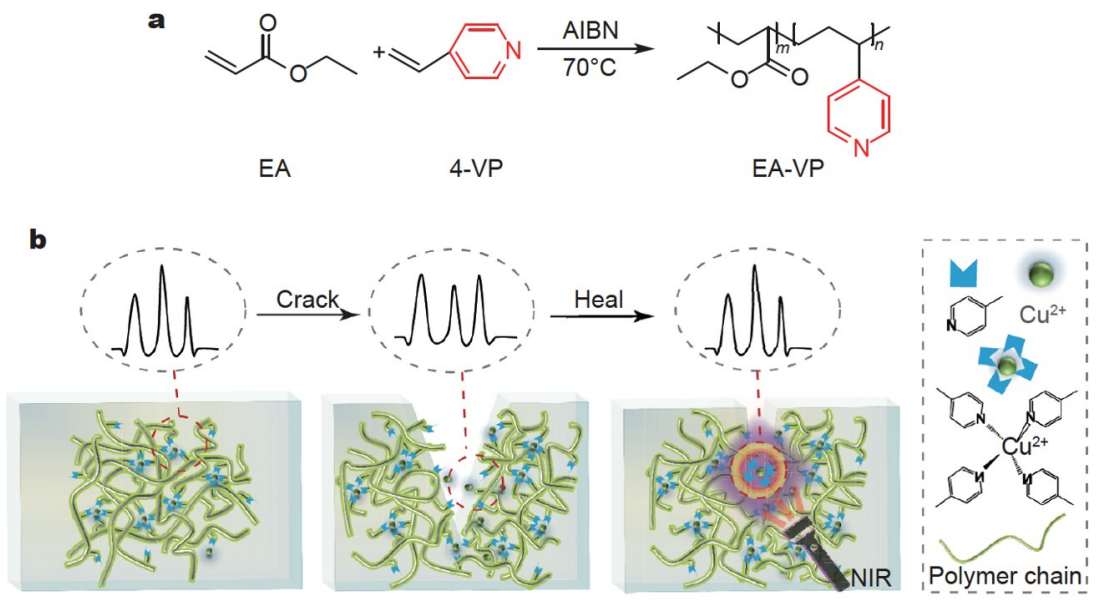

Figure 1 (a) Preparation process of the EA-VP elastomer. AIBN: 2,2-azobisisobutyronitrile. (b) Photothermal effect induced self-healing and Raman spectrum change of the pyridine- $\mathrm{Cu}$ dynamic bond in the process of self-healing. 

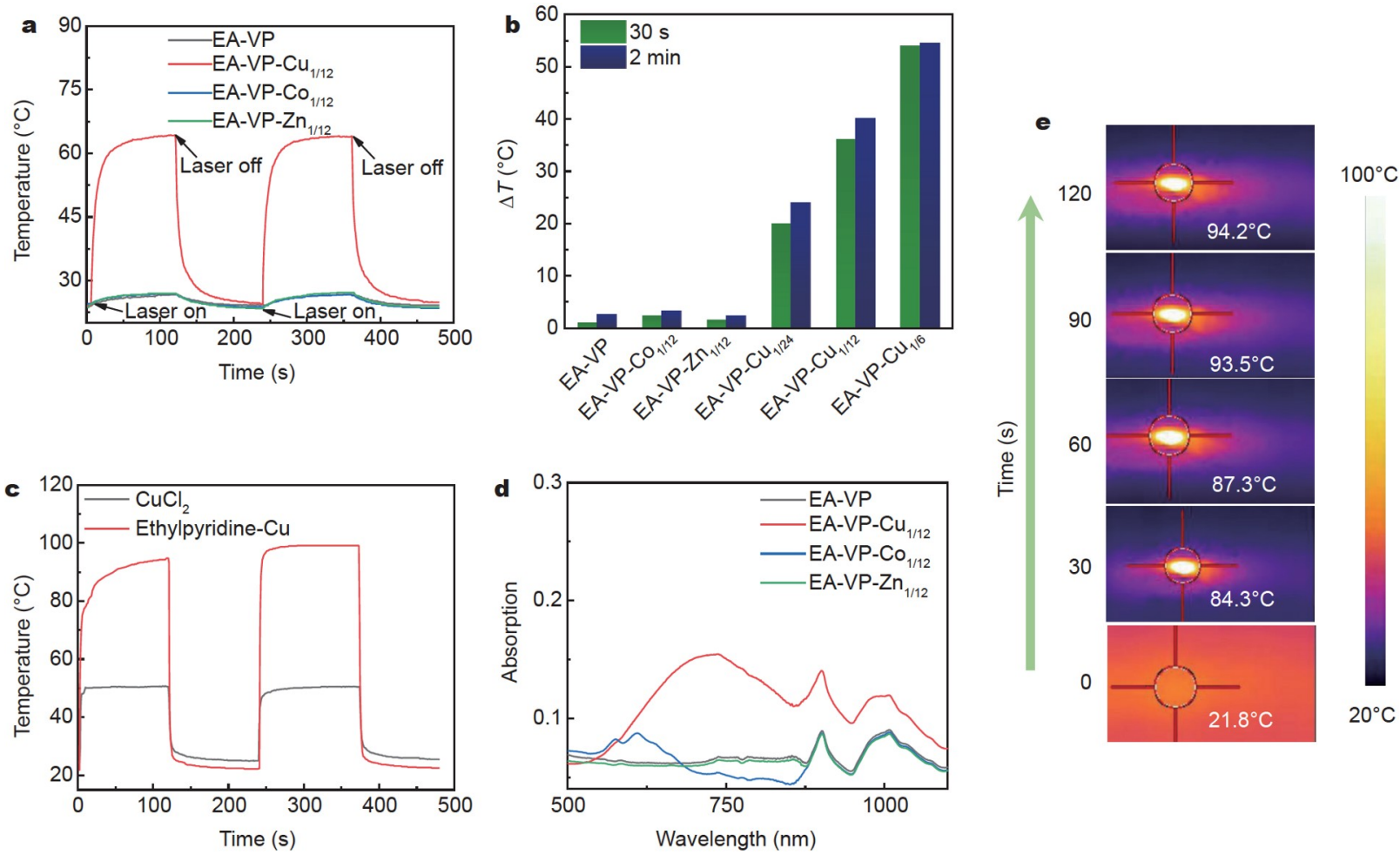

Figure 2 (a) Surface temperature profiles of EA-VP and EA-VP-M $\mathrm{M}_{x}$ upon exposure to a 785-nm laser, measured using the video mode of an infrared camera. (b) Temperature difference before and after laser irradiation for $30 \mathrm{~s}$ and 2 min of the EA-VP and EA-VP-M films. (c) Surface temperature profiles of the ethylpyridine-Cu complex and $\mathrm{CuCl}_{2}$ upon exposure to a 785-nm laser. (d) UV-vis-NIR absorption spectra of EA-VP and EA-VP-M ${ }_{x}$ (e) Infrared images of the ethylpyridine- $\mathrm{Cu}$ complex recorded using an infrared camera at different irradiation times.

the irradiation point rapidly increases from 24 to $60^{\circ} \mathrm{C}$ in $30 \mathrm{~s}$ and then gradually increases to $64^{\circ} \mathrm{C}$ in 2 min (Fig. 2a). Moreover, the temperature increment becomes more evident with increasing content of $\mathrm{Cu}^{2+}$ in the elastomer (Fig. 2b and Fig. S3). By contrast, the control experiments show that the temperature of the irradiation point only reaches $27^{\circ} \mathrm{C}$ for $\mathrm{EA}-\mathrm{VP}-\mathrm{Co}_{1 / 12}$ and EA-VP- $\mathrm{Zn}_{1 / 12}$ (Fig. 2a, b). These results demonstrate the superior photothermal effect of $\mathrm{Cu}^{2+}$ compared with other metal ions in the EA-VP copolymer.

In addition to the specific metal ion, the coordination with pyridine is also important. To illustrate this point, we measured the photothermal effect of a small-molecule model, $\mathrm{CuCl}_{2}$, and the ethylpyridine- $\mathrm{Cu}$ complex formed by blending ethylpyridine and $\mathrm{CuCl}_{2}$ with a molar ratio of $2: 1$. For pure $\mathrm{CuCl}_{2}$ powder with a high $\mathrm{Cu}^{2+}$ weight fraction of $47 \mathrm{wt} \%$, the temperature of the irradiation point only reaches $50^{\circ} \mathrm{C}$ in $30 \mathrm{~s}$ (Fig. 2c), and then hardly changes with an increase of irradiation time. This temperature increment is much lower than that of $\mathrm{EA}-\mathrm{VP}-\mathrm{Cu}_{1 / 12}$, containing only $0.4 \mathrm{wt} \%$ of $\mathrm{Cu}^{2+}$. On the contrary, for the ethylpyridine- $\mathrm{Cu}$ complex, the temperature of the irradiation point readily increases from 21.8 to $84.3^{\circ} \mathrm{C}$ within $30 \mathrm{~s}$ and then gradually rises to $94.2^{\circ} \mathrm{C}$ (Fig. $2 \mathrm{c}$, e). After the laser is turned off, the temperature rapidly drops to room temperature (Fig. 2c). This result indeed confirms that the pyridine- $\mathrm{Cu}$ coordination is the basic reason for the strong photothermal effect.

The basic mechanism governing the strong photothermal effect of EA-VP-Cu $\mathrm{C}_{x}$ is related to the near-infrared-light absorption and absence of fluorescence emission [21]. The light absorbance of EA-VP-M $\mathrm{M}_{x}$ was measured using a UV-vis-NIR spectrophotometer. We notice that the extinction coefficient of EA-VP-Cu $\mathrm{Cu}_{1 / 12}$ shows a strong dispersion peak from 550 to $860 \mathrm{~nm}$. However, this peak is absent in uncoordinated EA-VP, and the absorption peak of $\mathrm{CuCl}_{2}$ appears at $650-1130 \mathrm{~nm}$ (Fig. S4). This phenomenon indicates that the light absorption at a wavelength of $550-860 \mathrm{~nm}$ is enhanced after the formation of the pyridine- $\mathrm{Cu}$ coordination bonds (Fig. $2 \mathrm{~d}$ ). On the contrary, the individual absorption peaks of EA-VP-Co $\mathrm{Co}_{1 / 12}$ and EA-VP$\mathrm{Zn}_{1 / 12}$ are much smaller than that of EA-VP-Cu $\mathrm{Cu}_{1 / 12}$ (Fig. $2 \mathrm{~d}$ ), indicating the superior near-infrared light absorption of the pyridine- $\mathrm{Cu}$ coordination. Meanwhile, we also measured the fluorescence spectra of EA-VP-M $\mathrm{M}_{x}$. Although EA-VP-Cu $\mathrm{u}_{1 / 12}$ has a strong absorption at $550-860 \mathrm{~nm}$, it has no fluorescence emission (Fig. S5a), indicating that most of the absorbed light is converted into heat. In contrast, EA-VP- $\mathrm{Co}_{1 / 12}$ shows a strong fluorescence emission under the excitation of near-infrared light (Fig. S5b), indicating that the absorbed light energy is partly released in the form of fluorescence. With regard to EA-VP$\mathrm{Zn}_{1 / 12}$, emission and absorption are absent in the range of $500-850 \mathrm{~nm}$. These results indicate that the strong absorption and absence of fluorescence emission of EA-VP- $\mathrm{Cu}_{1 / 12}$ are the main reasons for its photothermal effect. Similar results are also observed in the small-molecule model of the ethylpyridine- $\mathrm{Cu}$ complex (Fig. S6).

\section{Mechanical and self-healing properties of the metallosupramolecular elastomer}

The mechanical performances of the polymers before and after coordination are examined by tensile tests. Fig. 3a shows the 

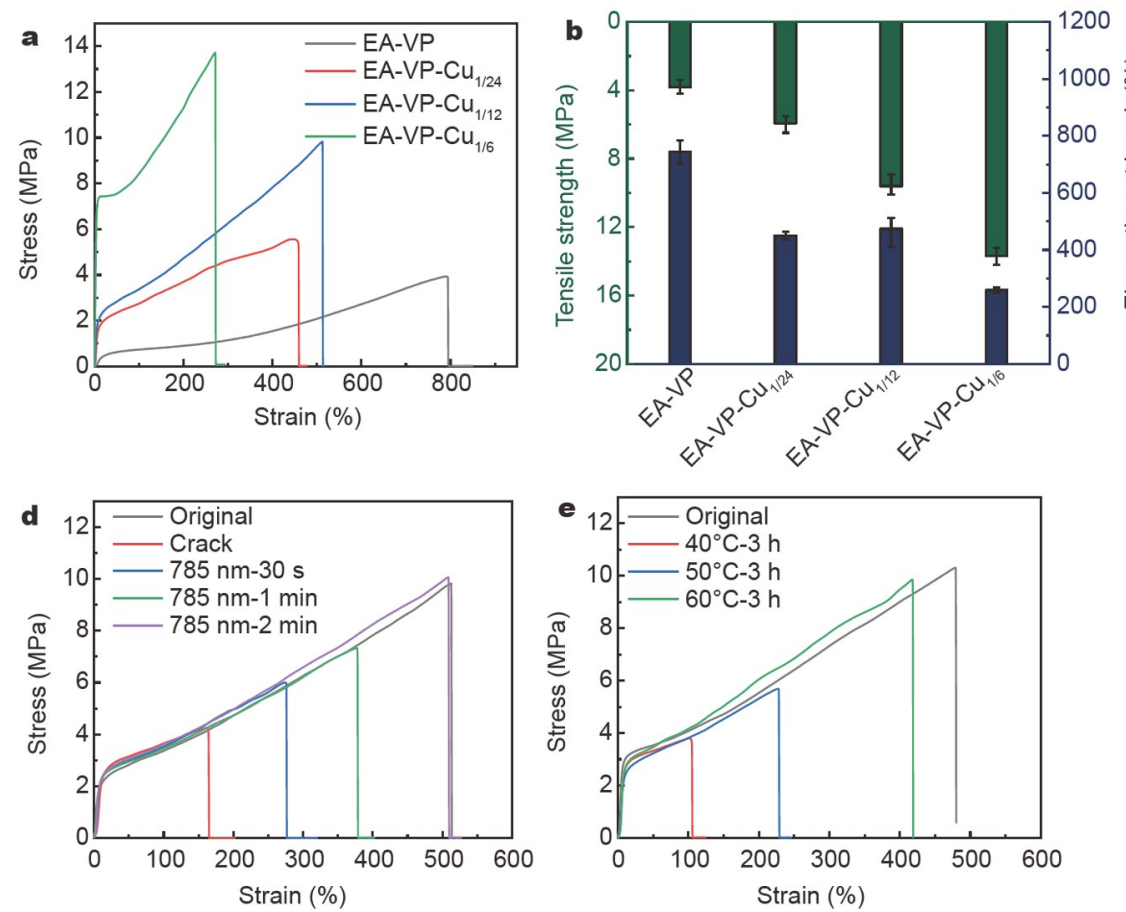
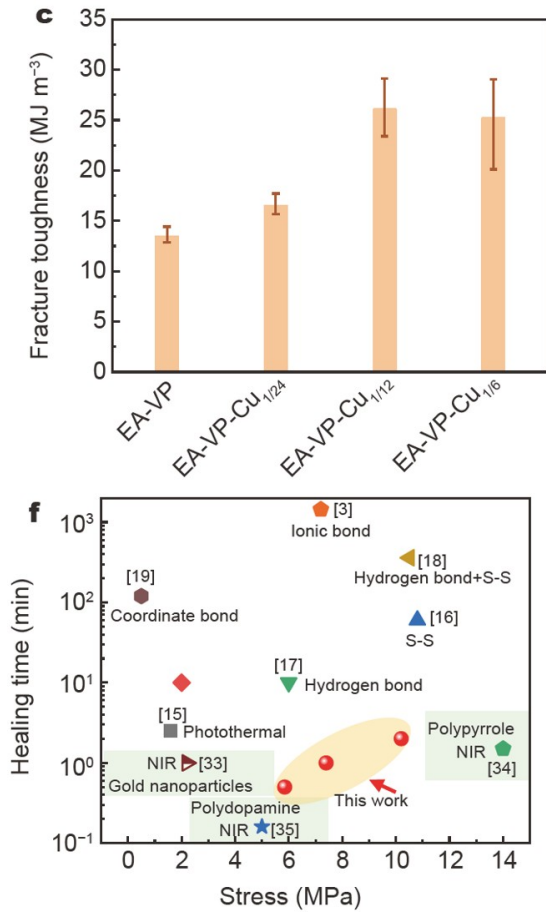

Figure 3 (a) Representative stress-strain curves of the EA-VP and EA-VP-Cu$u_{x}$ samples at a rate of $100 \mathrm{~mm} \mathrm{~min}^{-1}$. (b) Tensile strength and strain at break of EA-VP and EA-VP-Cu $x_{x}$ (c) Fracture toughness of EA-VP and EA-VP-Cu $x_{x}$ (d) Representative stress-strain curves of the uncut, scratched, and healed scratched samples under a 785-nm laser for different irradiation durations. (e) Representative stress-strain curves of the uncut and cut samples healed at 40 , 50 , and $60^{\circ} \mathrm{C}$ for $3 \mathrm{~h}$. (f) Ashby plot of the healing time needed versus tensile strength of EA-VP-Cu $\mathrm{u}_{1 / 12}$ and recently reported fast self-healing materials.

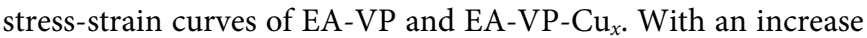
of $\mathrm{Cu}^{2+}$ loading, the dynamic crosslinking density gradually increases, leading to higher mechanical properties. The tensile stress and strain at break of EA-VP are $3.8 \pm 0.4 \mathrm{MPa}$ and $744 \%$ $\pm 43 \%$, respectively. As the pyridine $/ \mathrm{Cu}^{2+}$ molar ratio decreases to 6 , the tensile stress reaches $13.7 \pm 0.5 \mathrm{MPa}$, whereas the strain at break decreases to $259 \% \pm 10 \%$ (Fig. 3b). However, EA-VP$\mathrm{Cu}_{1 / 6}$ behaves like a tough plastic with a yielding point. By contrast, EA-VP-Cu $\mathrm{u}_{1 / 12}$ still shows an elastomeric behavior with long elongation and high fracture toughness (Fig. 3c). The effects of the metal-ligand coordination on mechanical strength are also clearly reflected by the difference in cyclic tensile behavior between EA-VP and EA-VP-Cu $\mathrm{1}_{1 / 12}$. The efficiency of energy dissipation is quantified as the area of the hysteresis loop divided by the area under the loading curve. The cyclic stressstrain curves of EA-VP-Cu $\mathrm{Cu}_{1 / 12}$ show larger hysteresis loops than that of EA-VP regardless of different maximum strains. The efficiency of the energy dissipation of EA-VP- $\mathrm{Cu}_{1 / 12}$ is $93 \%$ under $100 \%$ strain, which is much higher than the energy dissipation of $84 \%$ of the uncoordinated polymer (Fig. S7a, b). Therefore, introducing the pyridine-Cu coordination bonds significantly increases the energy dissipation of the elastomer, which contributes to the improved toughness of the elastomers (Fig. 3c).

To intuitively evaluate the self-healing process, a surface scratch of $30-50-\mu \mathrm{m}$ width induced by a razor blade on the EA$\mathrm{VP}-\mathrm{Cu}_{1 / 12}$ film is observed using a polarizing microscope. Results show that the scratch can be closed under heating at $40^{\circ} \mathrm{C}$ for $10 \mathrm{~min}$ (Fig. S8), but it closes in $20 \mathrm{~s}$ and almost vanishes in $2 \mathrm{~min}$ when heated to $60^{\circ} \mathrm{C}$ (Fig. 4a). Alternatively, at 785 -nm laser irradiation $\left(1.4 \mathrm{~W} \mathrm{~cm}^{-2}\right)$, the scratch almost closes within $20 \mathrm{~s}$ and vanishes after $2 \mathrm{~min}$ (Fig. 4b). Therefore, the healing process induced by infrared laser irradiation is similar to the repair process guided by thermal induction at $60^{\circ} \mathrm{C}$. Despite this fact, the self-healing process is more controllable and accessible by light irradiation than by ordinary thermal healing.

We also perform tensile tests to evaluate the self-healing properties of the scratched EA-VP- $\mathrm{Cu}_{1 / 12}$ samples and completely cut the samples. When a scratch is made on the sample, the tensile strength sharply decreases to $48 \%$ of the original condition. After being irradiated with a 785-nm laser for only $30 \mathrm{~s}$, the scratch is healed to $67 \%$ of the original value. It can be almost completely healed after irradiation for 2 min (Fig. 3d), which is faster than the healing time of most self-healing elastomers based on dynamic noncovalent bonds or photothermal fillers, such as polydopamine, polypyrrole, and gold nanoparticles, as illustrated in Fig. 3f [3,15-19,33-35]. Moreover, after $2 \mathrm{~min}$ of healing, the mechanical strength can be completely restored to the original value of $10 \mathrm{MPa}$, which is higher than most of the previously recorded values for fast self-healing polymers. To examine the healing properties of the completely cut samples at different temperatures, the two parts of the cut samples are then brought into contact. Stress-strain curves are measured after healing at 40,50 , and $60^{\circ} \mathrm{C}$ for $3 \mathrm{~h}$ and compared with those of the uncut sample, as shown in Fig. 3 e. At $40^{\circ} \mathrm{C}$, the sample shows a $37 \%$ recovery of tensile strength. The healing efficiency increases with increasing temperature and reaches $96 \%$ at $60^{\circ} \mathrm{C}$.

Molecular dynamics of the metallosupramolecular elastomer To understand the molecular dynamics underlying the fasthealing process, we used DSC and dynamic mechanical analysis 
(DMA) to investigate the influence of the pyridine- $\mathrm{Cu}$ coordination on the thermal and dynamic mechanical properties of EA-VP and EA-VP-Cu ${ }_{x}$ (Fig. 5). On the DSC heat flow curves, the glass transition temperature $\left(T_{\mathrm{gDSC}}\right)$ can be determined as the inflection point (Fig. 5a). $T_{\text {gDSC }}$ of EA-VP is $6.3^{\circ} \mathrm{C}$, and it increases to $10.3^{\circ} \mathrm{C}$ as the $\mathrm{Cu}^{2+}$ loading increases from $1 / 24$ to $1 / 6$ of the molar content of pyridine. It has been unraveled that DSC detects the enthalpic change accompanied by local segmental motion, which is the cooperative movement of neighboring short chains involving several repeat units [36]. The slight increase of $T_{\mathrm{gDSC}}$ indicates that the local segmental motion is slightly restrained by the supramolecular network formed by the pyridine- $\mathrm{Cu}$ coordination. From the temperature-dependent spectra of dynamic mechanical properties, we can also define three transition temperatures, $T_{E^{\prime}}, T_{E^{\prime \prime}}$, and $T_{\tan \delta}$, which represent the temperatures of the intersect of two tangent lines on the storage modulus $\left(E^{\prime}\right)$ curve (inset picture in Fig. 5b), peak of the loss modulus ( $\left.E^{\prime \prime}\right)$, and peak of $\tan \delta$, respectively (Fig. $5 \mathrm{~b}-\mathrm{d}$ ). Previous studies have revealed that $T_{E^{\prime}}$ and $T_{E^{\prime \prime}}$ have the same mechanism of molecular motion as $T_{\mathrm{gDSC}}$, i.e., local segmental motion [37,38]. Indeed, the $T_{E^{\prime}}$ and $T_{E^{\prime \prime}}$ values are very close to the $T_{\mathrm{gDSC}}$ values (Fig. $5 \mathrm{e}$ ), and their dependence on $\mathrm{Cu}^{2+}$ loading is almost the same. Such a phenomenon again confirms the slight restriction of the supramolecular network on the local segmental motion.

Unlike $T_{\mathrm{gDSC}}, T_{E^{\prime}}$, and $T_{E^{\prime \prime}}, T_{\tan \delta}$ is considerably higher (Fig. 5e). The reason for this phenomenon is that $T_{\tan \delta}$ arises from another mode of molecular motion, which has been explored by Plazek [39], Ngai [36], and Donth [40]. According to their studies, $T_{\tan \delta}$ can be attributed to the motion of entanglement constrained reptation modes in EA-VP. Our previous studies also support this conclusion $[38,41]$. Since the constrained reptation modes represent the motion of the Gaussian submolecules that involve 50 or more backbone bonds [36], they are entropic in nature and thus cannot be detected by DSC. However, the constrained reptation modes significantly contribute to the viscoelastic $\tan \delta$ peak measured by DMA. Therefore, $T_{\tan \delta}$ of EA-VP is much higher than that of local segmental motion (Fig. 5e). Moreover,
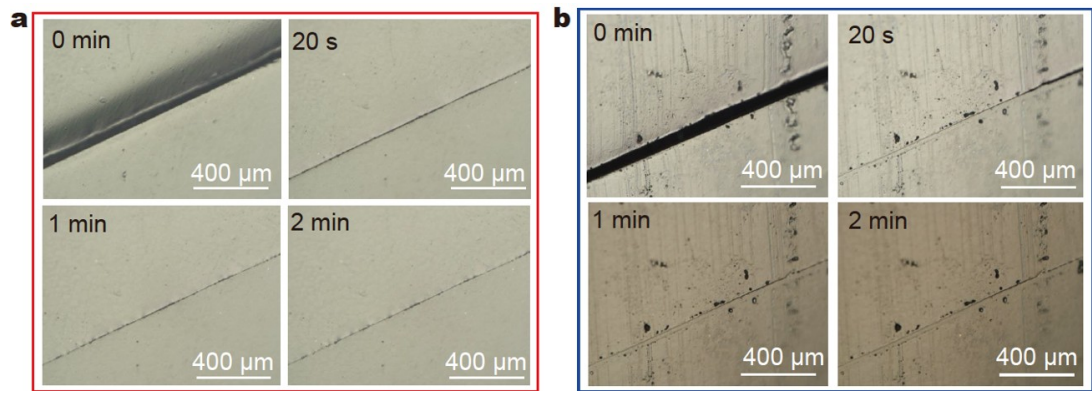

Figure 4 Optical microscopy images of the scratched EA-VP-Cu $\mathrm{Cu}_{1 / 12}$ film after healing (a) at $60^{\circ} \mathrm{C}$ and (b) under laser irradiation.
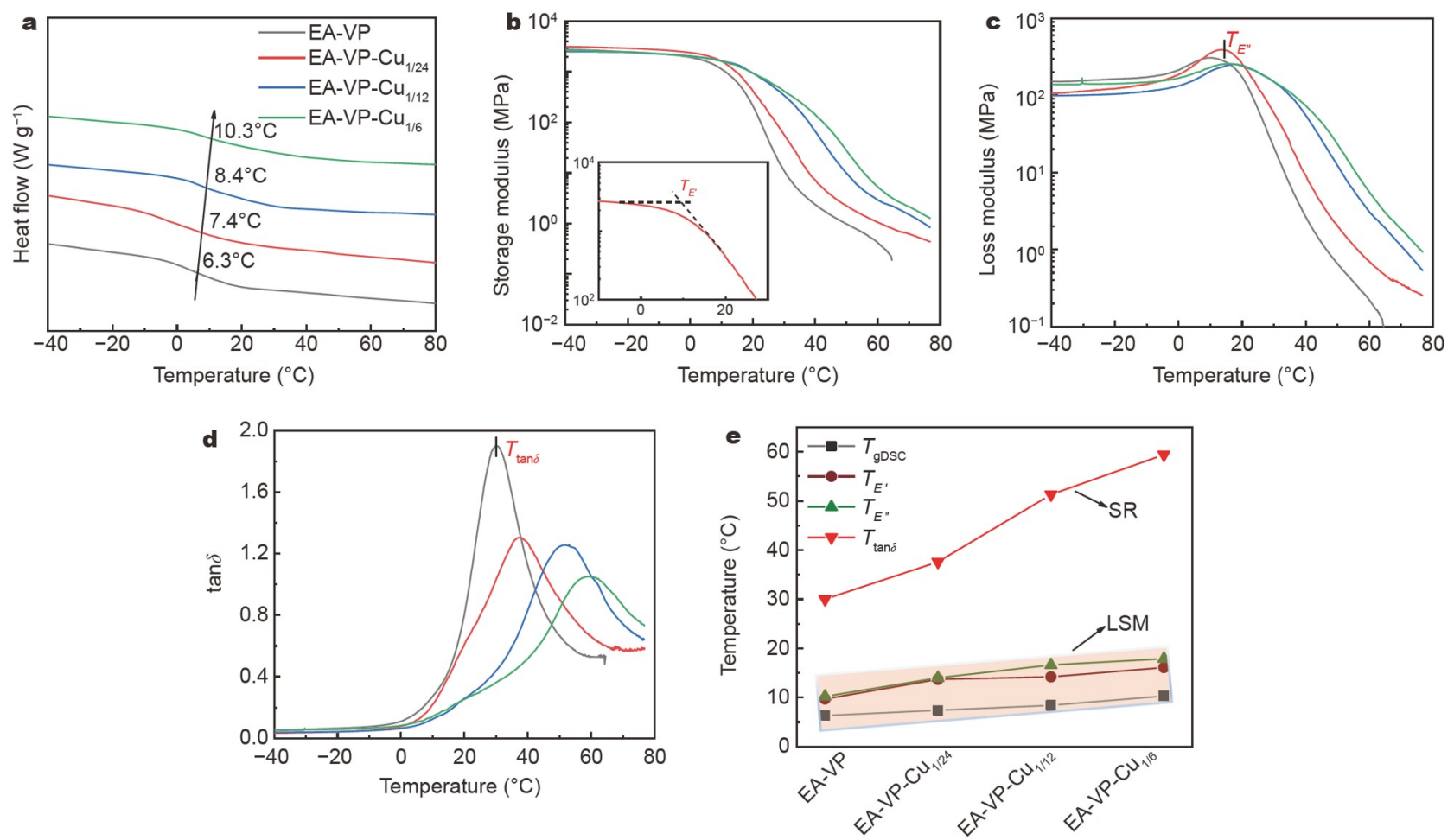

Figure 5 Thermal and dynamic mechanical properties. (a) DSC heat flow curves of EA-VP and EA-VP-Cu $x_{x}$. (b) Storage modulus curves, (c) loss modulus curves, (d) $\tan \delta$ curves of EA-VP and EA-VP-Cu $u_{x}$ (e) Different transition temperatures of EA-VP-Cu from DSC and DMA curves. 
upon introducing $\mathrm{Cu}^{2+}$, the constrained reptation modes are further restricted by the stickers formed by the pyridine- $\mathrm{Cu}$ coordination and thus become sticky reptation modes. Certainly, with an increase of $\mathrm{Cu}^{2+}$ loading, more stickers are formed and impose a stronger restriction of the sticky reptation modes. As such, $T_{\tan \delta}$ of EA-VP-Cu $\mathrm{Cu}_{x}$ remarkably increases with $\mathrm{Cu}^{2+}$ loading (Fig. 5e). When the molar ratio of pyridine $/ \mathrm{Cu}^{2+}$ is $6 / 1, T_{\tan \delta}$ of EA-VP-Cu $\mathrm{Cu}_{1 / 6}$ is increased by $35^{\circ} \mathrm{C}$ compared with that of EAVP. Therefore, the sticky reptation modes are more susceptible to the change of network structure than the local segmental motion. The underlying reason is that the Gaussian submolecules of sticky reptation modes are much longer than the local segments. This motion can be readily retarded by temporary crosslinking in a tubelike confinement formed by its neighbors [36]. From the DMA results of EA-VP-Cu $\mathrm{Cu}_{1 / 12}$, the relaxation temperatures of the local segmental motion and sticky reptation modes are 10 and $53^{\circ} \mathrm{C}$, respectively. Therefore, the local segmental motion is not strongly related with the selfhealing behavior. Instead, the major driving force of self-healing is sticky reptation modes. Only when the temperature is higher than that of sticky reptation modes, pyridine- $\mathrm{Cu}$ stickers are released, and the material can effectively undergo network reorganization to repair the mechanical damage.

Rheological measurements were conducted to explore the viscoelastic behaviors of EA-VP and EA-VP-Cu ${ }_{x}$. The master curve in Fig. 6a shows that the plateau modulus of EA-VP-Cu $\mathrm{Cu}_{1 / 12}$ is approximately twice that of EA-VP due to the presence of the temporary crosslinks generated by the pyridine- $\mathrm{Cu}$ supramolecular network. In addition, it should be noted that there are three intersections between the storage shear modulus $\left(G^{\prime}\right)$ and loss shear modulus $\left(G^{\prime \prime}\right)$ in the master curves. The first intersection occurs in the high-frequency region, corresponding to the local segmental motion of the glass transition. The frequencies of the first intersection of EA-VP and EA-VP-Cu $\mathrm{u}_{1 / 12}$ are almost similar (orange frame in Fig. 6a), again confirming that the coordination supramolecular network has a small effect on the local segmental motion. Despite this fact, the pyridine-Cu stickers evidently restrict the sticky reptation modes. Therefore, the second intersection of EA-VP-Cu $\mathrm{Cu}_{1 / 12}$ occurs at a lower frequency than that of EA-VP. The third intersection of EA-VP$\mathrm{Cu}_{1 / 12}$, where the viscous flow transition occurs, takes place at a lower frequency than that of EA-VP, revealing that the coordination interaction retards the terminal flow.

To discriminate the different modes of molecular motion, we acquire the relaxation spectrum over a wide time scale from the master curves of EA-VP and EA-VP-Cu $\mathrm{Cu}_{1 / 12}$ (see details in the Supplementary information). As shown in Fig. 6c, the local segmental motion takes place in the relaxation time range of $0.01 \mathrm{~s}<\tau<1 \mathrm{~s}$, and this range is nearly the same for EA-VP and EA-VP-Cu $\mathrm{Cu}_{1 / 12}$, as shown in the red rectangle frame in Fig. 6c. In contrast, the relaxation time range of sticky reptation modes becomes wider because the pyridine-Cu stickers restrict the reptation of molecular chains (black arrow represents EA-VP and red arrow represents $\left.\mathrm{EA}-\mathrm{VP}-\mathrm{Cu}_{1 / 12}\right)$. Meanwhile, the existence of the pyridine-Cu stickers also makes the terminal flow take longer time to occur. These results are consistent with the master curves and DMA curves, confirming that the pyridine$\mathrm{Cu}$ coordination exerts a stronger impact on the sticky reptation modes and terminal flow than the local segmental motion.

Fig. $6 \mathrm{~b}$ summarizes the logarithmic and natural logarithm values of the shift factor $\left(a_{T}\right)$ for the master curves. The relationship between $\lg a_{T}$ and $T-T_{s}$ can be well described by the Williams-Landel-Ferry (WLF) equation [36], where $T_{\mathrm{s}}$ is the reference temperature. EA-VP and EA-VP-Cu $\mathrm{Cu}_{1 / 12}$ show a very
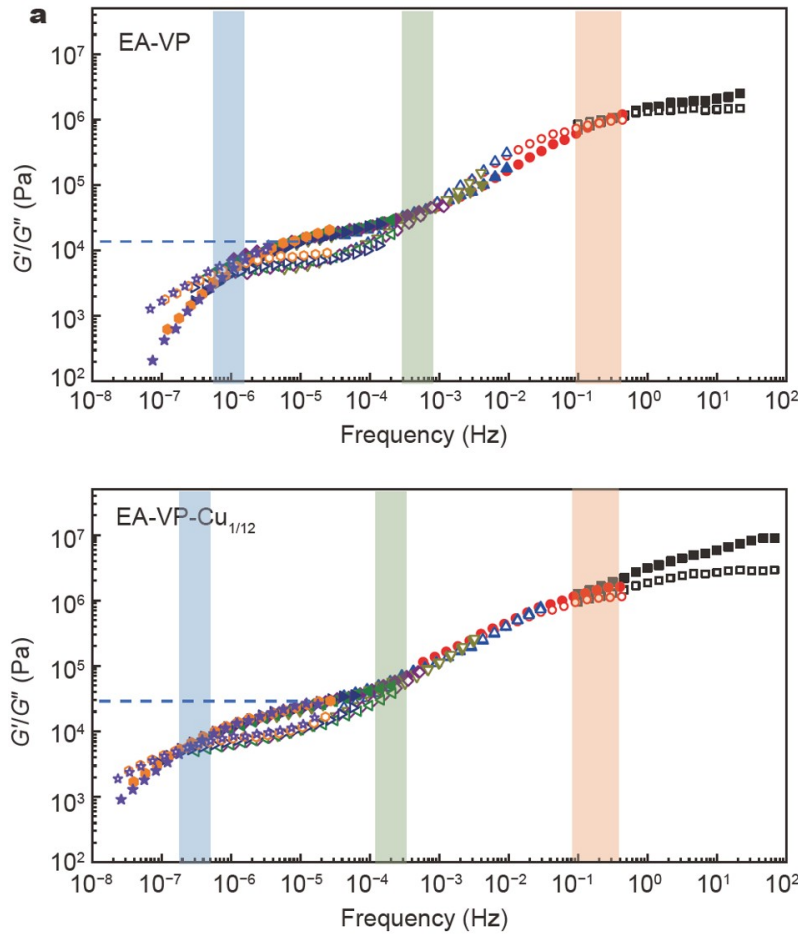

G' $G^{\prime \prime}$

- $283.15 \mathrm{~K}$

- $\circ 298.15 \mathrm{~K}$

• $\Delta 313.15 \mathrm{~K}$

$\nabla \quad \nabla 328.15 \mathrm{~K}$

- $\diamond 343.15 \mathrm{~K}$

$\triangleleft \triangleleft 358.15 \mathrm{~K}$

- $>373.15 \mathrm{~K}$

- $\quad 388.15 \mathrm{~K}$

* $\quad 403.15 \mathrm{~K}$
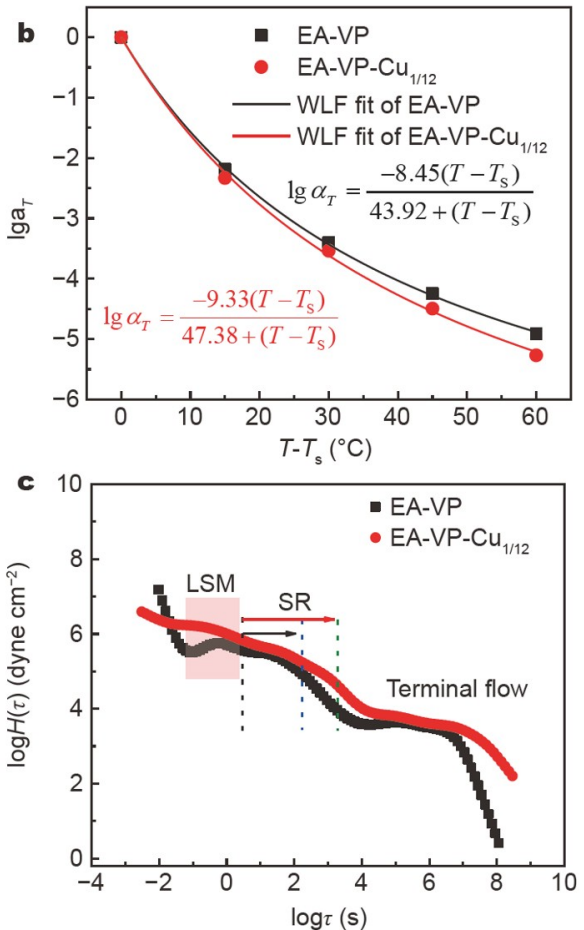

Figure 6 (a) Master curves of storage and loss moduli, $G^{\prime}$ and $G^{\prime \prime}$, as functions of angular frequency $\omega$ for $\mathrm{EA}-\mathrm{VP}$ and $\mathrm{EA}-\mathrm{VP}-\mathrm{Cu} \mathrm{u}_{1 / 12}$ at $T_{\mathrm{s}}=10^{\circ} \mathrm{C}$. (b) Temperature dependence of the translation factor of EA-VP and EA-VP-Cu $\mathrm{u}_{1 / 12}$, which can be described by the WLF equation. (c) Relaxation spectrum, plotted with logarithmic scales for EA-VP and EA-VP-Cu $\mathrm{Cu}_{1 / 12}$ at $T_{\mathrm{s}}=10^{\circ} \mathrm{C}$. 
similar temperature dependence of $\lg a_{T}$ around the $T_{\mathrm{g}}$. However, at temperatures remarkably higher than the glass transition temperature, $\lg a_{T}$ of EA-VP-Cu $\mathrm{Cu}_{1 / 12}$ shows a stronger temperature dependence than that of EA-VP. This again demonstrates that the stronger influence of the pyridine-Cu stickers on molecular motions is larger than local segments. At a higher temperature range of $85-130^{\circ} \mathrm{C}$, the relaxation behavior is dominated by the dissociation of stickers; thus, the shift factors reveal Arrhenius dependence, as shown in Fig. S9.

Break and reconstruction of coordination interaction revealed by micro-Raman spectroscopy

To unravel the bond-level healing kinetics, laser confocal microRaman spectroscopy was used to track the break and reconstruction of the coordination interaction during the damage and healing processes. First, ethylpyridine is selected as a smallmolecule model to study the change of the Raman spectrum after coordination. Fig. 7 displays the Raman spectra of pure ethylpyridine and the ethylpyridine- $\mathrm{CuCl}_{2}$ mixture (with a molar ratio of 120:1), which is the same as that in the coordination polymer. The Raman intensities and all vibrational frequency assignments of ethylpyridine and ethylpyridine-Cu complex are summarized in Table S1. The $\mathrm{C}-\mathrm{C}-\mathrm{C}$ (CCC) bending and $\mathrm{C}-\mathrm{N}-\mathrm{C}$ (CNC) bending/stretching of pure ethylpyridine are observed at $993 \mathrm{~cm}^{-1}$. After adding $\mathrm{CuCl}_{2}$, the peak intensity is obviously enhanced, which can be attributed to the formation of strong $\mathrm{Cu}-\mathrm{N}$ coordination bonds in the compound $[42,43]$. By contrast, the peak at $777 \mathrm{~cm}^{-1}$ representing the $\mathrm{H}-\mathrm{C}-\mathrm{C}-\mathrm{C}$ (HCCC) torsion vibration on the pyridine ring does not change before and after coordination. In pure ethylpyridine, the peak intensity ratio of $993-777 \mathrm{~cm}^{-1}\left(I_{993} / I_{777}\right)$ is 2.19 . However, when $\mathrm{CuCl}_{2}$ is added, the $I_{993} / I_{777}$ value becomes 2.61 .

We also studied the change of surface Raman spectrum of the EA-VP film upon adding $\mathrm{Cu}^{2+}$. It is found that the change is consistent with that of the small-molecule model. In the Raman spectrum of the EA-VP film, the CCC bending and CNC bending of the pyridine ring also appear at $993 \mathrm{~cm}^{-1}$, whereas the HCCC torsion vibration manifests a peak at $856 \mathrm{~cm}^{-1}$. The peak intensity ratio of $993-856 \mathrm{~cm}^{-1}\left(I_{993} / I_{856}\right)$ is 0.94 for the EAVP film (Fig. S10). However, this ratio increases to 1.54 for the EA-VP-Cu $\mathrm{Cu}_{1 / 12}$ film due to the formation of $\mathrm{Cu}-\mathrm{N}$ coordination bonds. Such a large difference can be used to track the microscopic damage and healing of the coordination bonds of the metallosupramolecular elastomer (Fig. 8).

For this purpose, a crack was made on the EA-VP-Cu $\mathrm{Cu}_{1 / 12}$ film, and Raman spectroscopy was used to record the spectrum

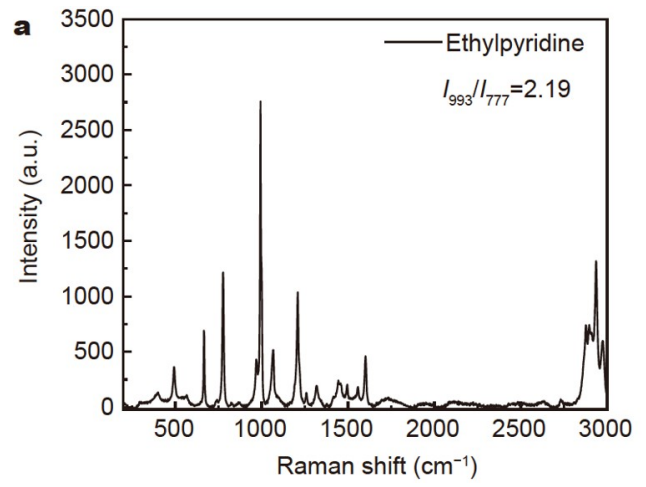

change for a point near the scratch during the damage and healing processes (note that this point is in the area activated by mechanical force). We can see that the peak intensity at $993 \mathrm{~cm}^{-1}$ of the point evidently decreases, and the $I_{993} / I_{856}$ value becomes much lower than that of the equilibrium and stable states. We define the decrease percentage (DP) as follows:

$\mathrm{DP}=\left(I_{993} / I_{856}\right.$ of equilibrium state $-I_{993} / I_{856}$ of nonequilibrium state $) /\left(I_{993} / I_{856}\right.$ of equilibrium state).

DP shows a sharp increase immediately after the scratch is generated. Such a high DP around the scratch reveals that the mechanical force destroys a part of the coordination bonds, leading to a nonequilibrium state. In order to examine the change of the coordination bonds in the self-healing process, we monitored the DP value as a function of the laser irradiation time at the mechanically activated point. Fig. $8 \mathrm{~b}$ displays that DP decreases with the extension of laser irradiation time and approaches $0 \%$ after 2 min of irradiation, demonstrating that light irradiation leads to the recovery of the coordination bonds. A control experiment on a point far away from the mechanically activated area of the EA-VP- $\mathrm{Cu}_{1 / 12}$ film, i.e., normal equilibrium surface, is also irradiated with a 785-nm laser under the same irradiation time (Fig. 8c). We can see that DP is close to $0 \%$ and remains almost unchanged with the extension of laser irradiation time (Fig. 8d). This phenomenon reveals that the photothermal effect has a negligible influence on the structure of the material in equilibrium.

The results observed above indicate that the break and reformation of the coordination bonds are strongly correlated with the mechanical damage and healing processes. When the blade induces a scratch on the surface of the EA-VP-Cu $\mathrm{Cu}_{1 / 12}$ film, a part of the coordination bonds are dissociated, and the molecular chains become metastable, forming a mechanically activated area around the scratch. Upon laser irradiation, the surface temperature reaches $60^{\circ} \mathrm{C}$, and thus, the mobility of sticky reptation modes is activated. This drives the metastable state of molecular chains to recover to an equilibrium state and the reformation of coordination bonds occurs, leading to the closure of the scratch.

\section{CONCLUSIONS}

In conclusion, we use pyridine- $\mathrm{Cu}$ coordination to fabricate a simple metallosupramolecular elastomer with high mechanical strength and fast self-healing ability. The pyridine- $\mathrm{Cu}$ coordination not only dynamically crosslinks the elastomer but also has a strong photothermal effect on the elastomer. Although the dynamic crosslinks only slightly retard the local segmental

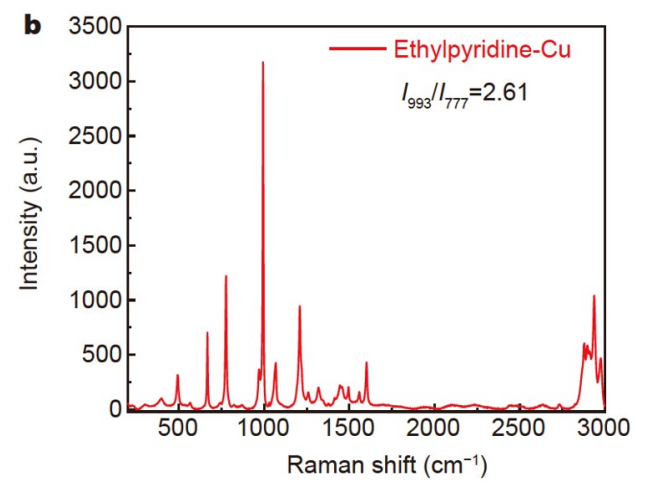

Figure 7 Raman spectra of the small molecule ethylpyridine (a) before and (b) after coordination. The molar ratio of ethylpyridine to copper ion is 120:1. 

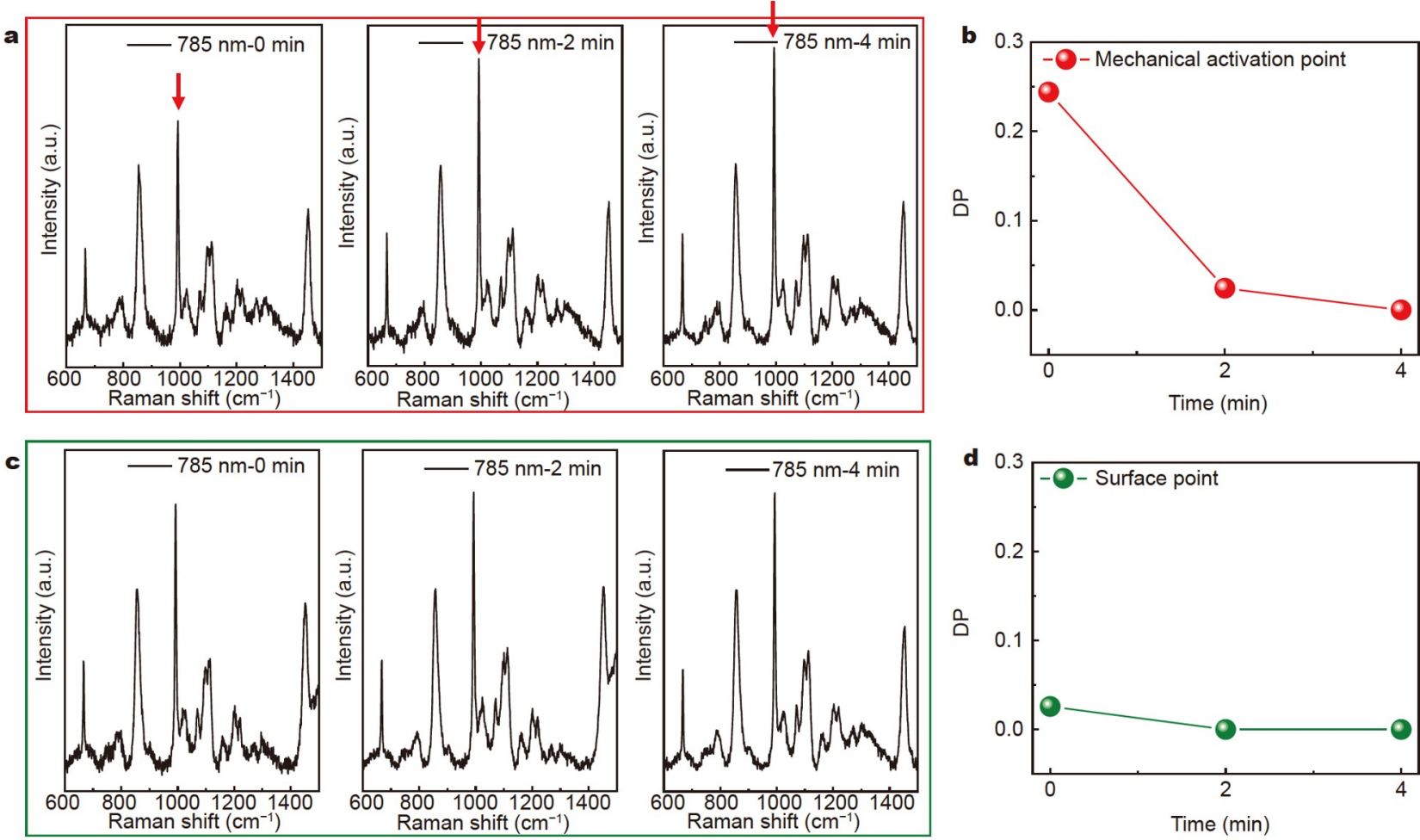

Figure 8 Fixed-point Raman spectroscopy of EA-VP-Cu $\mathrm{u}_{1 / 12}$ for the study of the self-healing mechanism. Raman spectra and DP of 785-nm laser irradiated for 0,2 , and 4 min: (a, b) on the surface and (c, d) during mechanical activation.

motion, they impose a strong restriction on the sticky reptation modes. Interestingly, the local segmental motion has less contribution to the healing efficiency, but the sticky reptation modes can act as the main driving force for self-healing. As such, the mechanically damaged elastomer can be readily healed under 785-nm near-infrared light as the sticky reptation modes are activated by the photothermal effect, which raises the temperature to more than $60^{\circ} \mathrm{C}$. Moreover, the break and reformation of the coordination bonds can be sensitively detected by the changes in the micro-Raman spectra. This enables us to track the bond-level kinetics during the self-healing process. Therefore, this work not only provides a facile route for designing novel self-healing elastomers but also points out a method to quantitatively monitor the nonequilibrium state in the failure region.

\section{Received 19 November 2021; accepted 7 January 2022; published online 18 February 2022}

1 Wang $\mathrm{H}$, Liu $\mathrm{H}$, Cao Z, et al. Room-temperature autonomous selfhealing glassy polymers with hyperbranched structure. Proc Natl Acad Sci USA, 2020, 117: 11299-11305

2 Mozhdehi D, Ayala S, Cromwell OR, et al. Self-healing multiphase polymers via dynamic metal-ligand interactions. J Am Chem Soc, 2014, 136: $16128-16131$

3 Peng Y, Zhao L, Yang C, et al. Super tough and strong self-healing elastomers based on polyampholytes. J Mater Chem A, 2018, 6: 1906619074

4 Sinawang G, Osaki M, Takashima Y, et al. Supramolecular self-healing materials from non-covalent cross-linking host-guest interactions. Chem Commun, 2020, 56: 4381-4395

5 Wu P, Cheng $\mathrm{H}$, Wang $\mathrm{X}$, et al. A self-healing and recyclable polyurethane-urea Diels-Alder adduct synthesized from carbon dioxide and furfuryl amine. Green Chem, 2021, 23: 552-560

6 Huang S, Podgórski M, Han X, et al. Chemical recycling of poly (thiourethane) thermosets enabled by dynamic thiourethane bonds. Polym Chem, 2020, 11: 6879-6883

7 Xiao G, Wang Y, Zhang H, et al. Facile strategy to construct a selfhealing and biocompatible cellulose nanocomposite hydrogel via reversible acylhydrazone. Carbohydr Polyms, 2019, 218: 68-77

8 Wang Y, Chen Q, Chen M, et al. PHEMA hydrogel films crosslinked with dynamic disulfide bonds: Synthesis, swelling-induced mechanical instability and self-healing. Polym Chem, 2019, 10: 4844-4851

9 Zhang W, Wu B, Sun S, et al. Skin-like mechanoresponsive self-healing ionic elastomer from supramolecular zwitterionic network. Nat Commun, 2021, 12: 4082

10 Bai L, Zheng J. Robust, reprocessable and shape-memory vinylogous urethane vitrimer composites enhanced by sacrificial and self-catalysis Zn(II)-ligand bonds. Compos Sci Tech, 2020, 190: 108062

11 Wang W, Wang F, Zhang C, et al. Robust, reprocessable, and reconfigurable cellulose-based multiple shape memory polymer enabled by dynamic metal-ligand bonds. ACS Appl Mater Interfaces, 2019, 12: 25233-25242

12 Yanagisawa Y, Nan Y, Okuro K, et al. Mechanically robust, readily repairable polymers via tailored noncovalent cross-linking. Science, 2018, 359: 72-76

13 Zhang L, Liu Z, Wu X, et al. A highly efficient self-healing elastomer with unprecedented mechanical properties. Adv Mater, 2019, 31: 1901402

14 Wu S, Fang S, Tang Z, et al. Bioinspired design of elastomeric vitrimers with sacrificial metal-ligand interactions leading to supramechanical robustness and retentive malleability. Mater Des, 2020, 192: 108756

15 Chen Y, Zhao X, Luo C, et al. A facile fabrication of shape memory polymer nanocomposites with fast light-response and self-healing performance. Compos Part A-Appl Sci Manuf, 2020, 135: 105931

$16 \mathrm{Wu} \mathrm{H}, \mathrm{Xie} \mathrm{H}$, Tian $\mathrm{X}$, et al. Hard, tough and fast self-healing thermoplastic polyurethane. Prog Org Coatings, 2021, 159: 106409

17 Yan J, Li M, Wang Z, et al. Highly tough, multi-stimuli-responsive, and fast self-healing supramolecular networks toward strain sensor application. Chem Eng J, 2020, 389: 123468

18 Ying WB, Yu Z, Kim DH, et al. Waterproof, highly tough, and fast self- 
healing polyurethane for durable electronic skin. ACS Appl Mater Interfaces, 2020, 12: 11072-11083

19 Yang J, Zhang Z, Yan Y, et al. Highly stretchable and fast self-healing luminescent materials. ACS Appl Mater Interfaces, 2020, 12: 1323913247

20 Li CH, Zuo JL. Self-healing polymers based on coordination bonds. Adv Mater, 2019, 32: 1903762

21 Burnworth M, Tang L, Kumpfer JR, et al. Optically healable supramolecular polymers. Nature, 2011, 472: 334-337

22 Habault D, Zhang H, Zhao Y. Light-triggered self-healing and shapememory polymers. Chem Soc Rev, 2013, 42: 7244-7256

23 Neumann LN, Oveisi E, Petzold A, et al. Dynamics and healing behavior of metallosupramolecular polymers. Sci Adv, 2021, 7: eabe4154

24 Kang J, Son D, Wang GJN, et al. Tough and water-insensitive selfhealing elastomer for robust electronic skin. Adv Mater, 2018, 30: 1706846

25 Qiu X, Guo Q, Wang Y, et al. Self-healing and reconfigurable actuators based on synergistically cross-linked supramolecular elastomer. ACS Appl Mater Interfaces, 2020, 12: 41981-41990

26 Feiteira J, Tsangouri E, Gruyaert E, et al. Monitoring crack movement in polymer-based self-healing concrete through digital image correlation, acoustic emission analysis and SEM in-situ loading. Mater Des, 2017, 115: 238-246

27 Shen Q, Liu H, Peng Y, et al. Visualization of the self-healing process by directly observing the evolution of fluorescence intensity. Polym Chem, 2021, 12: 494-500

28 Xia NN, Xiong XM, Rong MZ, et al. Self-healing of polymer in acidic water toward strength restoration through the synergistic effect of hydrophilic and hydrophobic interactions. ACS Appl Mater Interfaces, 2017, 9: 37300-37309

29 Geitner R, Legesse FB, Kuhl N, et al. Do you get what you see? Understanding molecular self-healing. Chem Eur J, 2018, 24: 2493-2502

30 Zhang L, Wang $\mathrm{H}$, Zhu Y, et al. Electron-donating effect enabled simultaneous improvement on the mechanical and self-healing properties of bromobutyl rubber ionomers. ACS Appl Mater Interfaces, 2020, 12: $53239-53246$

31 Davydovich D, Urban MW. Water accelerated self-healing of hydrophobic copolymers. Nat Commun, 2020, 11: 1-7

32 Andreozzi L, Castelvetro V, Faetti M, et al. Rheological and thermal properties of narrow distribution poly(ethyl acrylate)s. Macromolecules, 2006, 39: 1880-1889

33 Zhang K, Zhang J, Liu Y, et al. A NIR laser induced self-healing PDMs/ gold nanoparticles conductive elastomer for wearable sensor. J Colloid Interface Sci, 2021, 599: 360-369

$34 \mathrm{Wu} \mathrm{H}$, Sheng D, Liu X, et al. NIR induced self-healing polyurethane/ polypyrrole nanocomposites. Polymer, 2020, 189: 122181

35 Yang L, Wang Z, Fei G, et al. Polydopamine particles reinforced poly (vinyl alcohol) hydrogel with NIR light triggered shape memory and self-healing capability. Macromol Rapid Commun, 2017, 38: 1700421

36 Ngai KL, Plazek DJ. Identification of different modes of molecular motion in polymers that cause thermorheological complexity. Rubber Chem Tech, 1995, 68: 376-434

$37 \mathrm{Wu}$, Huang G, Wang X, et al. Changes in the viscoelastic mechanisms of polyisobutylene by plasticization. Macromolecules, 2012, 45: 80518057

38 Lei Z, Xing W, Wu J, et al. The proper glass transition temperature of amorphous polymers on dynamic mechanical spectra. J Therm Anal Calorim, 2014, 116: 447-453

39 Plazek DJ, Chay IC, Ngai KL, et al. Viscoelastic properties of polymers. 4. Thermorheological complexity of the softening dispersion in polyisobutylene. Macromolecules, 1995, 28: 6432-6436

40 Donth E, Beiner M, Reissig S, et al. Fine structure of the main transition in amorphous polymers: Entanglement spacing and characteristic length of the glass transition. Discussion of examples. Macromolecules, 1996, 29: 6589-6600

$41 \mathrm{Wu}$ J, Huang G, Qu L, et al. Correlations between dynamic fragility and dynamic mechanical properties of several amorphous polymers. J NonCryst Solids, 2009, 355: 1755-1759

42 Gökce H, Bahçeli S. A study of molecular structure and vibrational spectra of copper(II) halide complex of 2-(2'-thienyl)pyridine. Spectrochim Acta Part A-Mol Biomol Spectr, 2012, 96: 139-147

43 Gökce H, Bahçeli S. FT-IR, micro-Raman and UV-vis spectroscopic and quantum chemical investigations of free $2,2^{\prime}$-dithiodipyridine and its metal ( $\mathrm{Co}, \mathrm{Cu}$ and $\mathrm{Zn}$ ) halide complexes. Spectrochim Acta Part AMol Biomol Spectr, 2013, 114: 61-73

Acknowledgements This work was supported by the National Natural Science Foundation of China (51873110), Sichuan Science and Technology Program (2021JDJQ0018), and the State Key Laboratory of Polymer Materials Engineering.

Author contributions $\quad \mathrm{Liu} \mathrm{H}$ and $\mathrm{Wu} \mathrm{J}$ designed and engineered the samples; Liu H and Shen Q performed the experiments; Liu H wrote the paper with support from $\mathrm{Wu}$ J. All authors contributed to the general discussion.

Conflict of interest The authors declare that they have no conflict of interest.

Supplementary information Experimental details and supporting data are available in the online version of the paper.

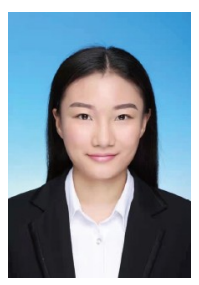

Hui Liu received a bachelor's degree from the College of Materials Science and Engineering at the Wuhan University of Technology in 2016 and a master's degree from the Academy of Aerospace Solid Propulsion Technology in 2019. She is currently pursuing her $\mathrm{PhD}$ degree at $\mathrm{Si}$ chuan University, under the supervision of Prof. Wu. Her research interests focus on the design and preparation of self-healing elastomeric materials and self-healing mechanisms.

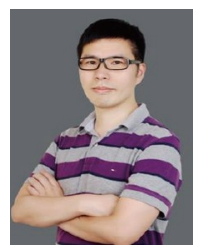

Jinrong Wu is a professor at the College of Polymer Science and Engineering, Sichuan University. He received a bachelor's degree and a doctoral degree in 2003 and 2008 , respectively, from the College of Polymer Science and Engineering, Sichuan University. He studied at Texas Tech University as a visiting student from 2007 to 2008 and at Harvard University as a visiting scholar from 2014 to 2016. His current research involves high-performance, functional, and self-healing elastomers and related theoretical problems of elastomer materials.

\section{基于吡啶-铜配位的快速自修复高性能金属超分子弹 性体}

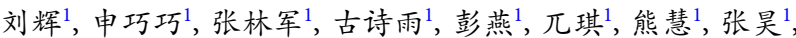

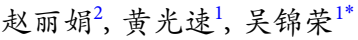

摘要 在机械力破坏下, 弹性体容易出现裂纹, 而裂纹若没有及时被修 复, 容易引发灾难性事故. 因此, 对弹性体来说, 具有快速修复裂纹的能 力非常重要. 然而, 开发同时具有快速修复能力和高机械性能的弹性体 极具挑战性. 本论文中, 我们将吡啶-铜配位键引入丙烯酸乙酯和乙烯 基吡啶的共聚物中来制备金属配位弹性体. 由于吡啶-铜配位键具有强 烈的光热效应, 在近红外光照射 $30 \mathrm{~s}$ 后, 样品表面温度可以从 $24^{\circ} \mathrm{C}$ 升高 至 $60^{\circ} \mathrm{C}$, 在 $60^{\circ} \mathrm{C}$ 时, sticky-reptation运动被激活, 为金属超分子弹性体网 络重组和快速自修复提供驱动力. 材料拉伸强度可达到 $10 \mathrm{MPa}$, 并且划 痕破坏试样和完全断裂破坏的试样可分别在 $2 \mathrm{~min}$ 和 $3 \mathrm{~h}$ 内愈合. 此外, 在破坏和修复过程中，激光共焦显微拉曼光谱可以追踪配位键的断裂 和重组, 这为监测金属超分子聚合物的键级修复动力学提供了一种微 观方法. 